Revista de Matemática: Teoría y Aplicaciones 2009 16(1) : 127-136

CIMPA - UCR ISSN: 1409-2433

\title{
MINIMIZATION OF THE FIRST EIGENVALUE IN PROBLEMS INVOLVING THE BI-LAPLACIAN
}

\author{
Claudia Anedda* Fabrizio CucCu $^{\dagger}$ Giovanni Porru ${ }^{\ddagger}$
}

Recibido/Received: 20 Feb 2008 - Aceptado/Accepted: 25 Jul 2008

\begin{abstract}
This paper concerns the minimization of the first eigenvalue in problems involving the bi-Laplacian under either homogeneous Navier boundary conditions or homogeneous Dirichlet boundary conditions. Physically, in case of $N=2$, our equation models the vibration of a non homogeneous plate $\Omega$ which is either hinged or clamped along the boundary. Given several materials (with different densities) of total extension $|\Omega|$, we investigate the location of these materials inside $\Omega$ so to minimize the first mode in the vibration of the corresponding plate.
\end{abstract}

Keywords: bi-Laplacian, first eigenvalue, minimization.

\section{Resumen}

Este artículo trata de la minimización del primer autovalor en problemas relativos al bi-Laplaciano bajo condiciones de frontera homogéneas de tipo Navier o Dirichlet. Físicamente, en el problema bi-dimensional, nuestra ecuacin modela la vibración de una placa inhomogénea $\Omega$ fija con goznes a lo largo de su borde. Dados varios materiales (de diferentes densidades) y extensión total $|\Omega|$, investigamos cuál debe ser la localización de tales materiales en la placa para minimizar el primer modo de su vibración.

Palabras clave: bi-Laplaciano, primer autovalor, minimización.

Mathematics Subject Classification: 35P15, 47A75, 49K20.

\footnotetext{
*Dipartimento di Matematica e Informatica, Universitá di Cagliari Via Ospedale 72, 09124 Cagliari, Italy. E-Mail: canedda@unica.it

${ }^{\dagger}$ Misma dirección que C. Anedda. E-Mail: fcuccu@unica.it

${ }^{\ddagger}$ Misma dirección que C. Anedda. E-Mail: porru@unica.it
} 


\section{Introduction}

Let $\Omega$ be a bounded smooth domain in $\mathbb{R}^{N}$ and let $g_{0}$ be a measurable function satisfying $0 \leq g_{0} \leq M$ in $\Omega$, where $M$ is a positive constant. To avoid trivial situations, we always assume $g_{0} \not \equiv 0$ and $g_{0} \not \equiv M$. Define $\mathcal{G}$ as the family of all measurable functions defined in $\Omega$ which are rearrangements of $g_{0}$. Consider the following eigenvalue problems

$$
\Delta^{2} u=\lambda g u, \quad \text { in } \quad \Omega, \quad u=\Delta u=0 \quad \text { on } \quad \partial \Omega,
$$

and

$$
\Delta^{2} v=\Lambda g v, \quad \text { in } \quad \Omega, \quad v=\frac{\partial v}{\partial \nu}=0 \quad \text { on } \quad \partial \Omega,
$$

where $g \in \mathcal{G}, \lambda=\lambda_{g}, \Lambda=\Lambda_{g}$ are the first eigenvalues and $u, v$ are the corresponding eigenfunctions. The operator $\Delta^{2}$ stands for the usual bi-Laplacian, that is $\Delta^{2} u=\Delta(\Delta u)$. The first eigenvalue $\lambda$ of problem (1) is obtained by minimizing the associate Rayleigh quotient

$$
\lambda=\inf \left\{\frac{\int_{\Omega}(\Delta z)^{2} d x}{\int_{\Omega} g z^{2} d x}: \quad z, \Delta z \in H_{0}^{1}(\Omega), \quad z \not \equiv 0\right\} .
$$

The first eigenvalue $\Lambda$ of problem (2) is obtained by minimizing the quotient

$$
\Lambda=\inf \left\{\frac{\int_{\Omega}(\Delta z)^{2} d x}{\int_{\Omega} g z^{2} d x}: \quad z \in H_{0}^{2}(\Omega), \quad z \not \equiv 0\right\} .
$$

It is well known that the inferior is attained in both cases [14]. The minimum of (3) satisfies problem (1) in the weak sense, that is

$$
\int_{\Omega} \Delta u \Delta z d x=\lambda \int_{\Omega} g u z d x, \quad \forall z: z, \Delta z \in H_{0}^{1}(\Omega) .
$$

The minimum of (4) satisfies problem (2) in the sense

$$
\int_{\Omega} \Delta v \Delta z d x=\Lambda \int_{\Omega} g v z d x, \quad \forall z \in H_{0}^{2}(\Omega) .
$$

By regularity results (see [1]) the solutions to problems (1) and (2) belong to $H_{l o c}^{4}(\Omega)$.

In this paper we investigate the problems

$$
\min _{g \in \mathcal{G}} \lambda_{g}, \quad \text { and } \quad \min _{g \in \mathcal{G}} \Lambda_{g} .
$$

Let us give a motivation for the study of these problems in case of $N=2$. Physically, our equations model the vibration of a non homogeneous plate $\Omega$ which is either hinged or clamped along the boundary $\partial \Omega$. Given several materials (with different densities) of total extension $|\Omega|$, we investigate the location of these materials inside $\Omega$ so to minimize the first mode in the vibration of the plate. The corresponding problem for second order equations has been discussed in several papers, see for example [6], [7], [9].

The paper is organized as follows. In Section 2 we collect some definitions and known results. In Section 3 we investigate the problems (5) proving results of existence and results of representation of minimizers. In case $\Omega$ is a ball we prove uniqueness for both problems. 


\section{Preliminaries}

Denote with $|E|$ the Lebesgue measure of the (measurable) set $E \subset \mathbb{R}^{N}$. Given a measurable function $g_{0}(x)$ defined in $\Omega$ we say that $g(x)$, defined in $\Omega$, belongs to the class of rearrangements $\left.\mathcal{G}=\mathcal{G}(\}_{\prime}\right)$ if $|\{x \in \Omega: g(x) \geq \beta\}|=\left|\left\{x \in \Omega: g_{0}(x) \geq \beta\right\}\right| \quad \forall \beta \in \mathbb{R}$.

We make use of the following results.

Lemma 2.1 Let $g \in L^{1}(\Omega)$ and let $u \in L^{1}(\Omega)$. Suppose that every level set of $u$ (that is, sets of the form $\left.u^{-1}(\{\alpha\})\right)$, has measure zero. Then there exists an increasing function $\phi$ such that $\phi(u)$ is a rearrangement of $g$.

Proof. The assertion follows by Lemma 2.9 of [4].

Lemma 2.2 Let $\mathcal{G}$ be the set of rearrangements of a fixed function $g_{0} \in L^{r}(\Omega), r>1$, $g_{0} \not \equiv 0$, and let $\overline{\mathcal{G}}$ denote the weak closure of $\mathcal{G}$ in $L^{r}(\Omega)$. If $u \in L^{s}(\Omega), s=r /(r-1)$, $u \not \equiv 0$, and if there is an increasing function $\phi$ such that $\phi(u) \in \mathcal{G}$ then

$$
\int_{\Omega} g u d x \leq \int_{\Omega} \phi(u) u d x \quad \forall g \in \overline{\mathcal{G}}
$$

and the function $\phi(u)$ is the unique maximizer relative to $\overline{\mathcal{G}}$.

Proof. The assertion follows by Lemma 2.4 of [4].

Lemma 2.3 Let $\mathcal{G}$ be the set of rearrangements of a fixed function $g_{0} \in L^{r}(\Omega), r>1$, $g_{0} \not \equiv 0$, and let $u \in L^{s}(\Omega), s=r /(r-1), u \not \equiv 0$. There exists $\bar{g} \in \mathcal{G}$ such that

$$
\int_{\Omega} g u d x \leq \int_{\Omega} \bar{g} u d x \quad \forall g \in \overline{\mathcal{G}} .
$$

Proof. It follows by Lemma 2.4 of [4]. See also [5].

Next we recall a well known rearrangement inequality. For $u$ non negative in $\Omega, u^{\sharp}$ denotes the decreasing Schwarz rearrangement of $u$; that is, $u^{\sharp}$ is defined in $\Omega^{\sharp}$, the ball centered in the origin with measure equal to $|\Omega|$, is radially symmetric, decreases as $|x|$ increases, and satisfies

$$
|\{x \in \Omega: u(x) \geq \beta\}|=\left|\left\{x \in \Omega^{\sharp}: u^{\sharp}(x) \geq \beta\right\}\right| \quad \forall \beta \geq 0 .
$$

If $u \in H_{0}^{1}(\Omega)$ is non-negative and if $u^{\sharp}$ is the decreasing Schwarz rearrangement of $u$ then $u^{\sharp} \in H_{0}^{1}\left(\Omega^{\sharp}\right)$ and the inequality

$$
\int_{\Omega^{\sharp}}\left|\nabla u^{\sharp}\right|^{2} d x \leq \int_{\Omega}|\nabla u|^{2} d x
$$

holds. The case of equality in (6) has been considered in [3]. We have

Lemma 2.4 Let $u \in H_{0}^{1}(\Omega)$ be non-negative, and suppose equality holds in (6). If

$$
\left|\left\{x \in \Omega^{\sharp}: \nabla u^{*}(x)=0, \quad 0<u^{*}(x)<\sup _{\Omega} u(x)\right\}\right|=0
$$

then $u$ is a translate of $u^{\sharp}$.

Proof. See Theorem 1.1 of [3] or the monograph [13]. 


\section{Main results}

Let $\Omega \subset \mathbb{R}^{N}$ be a bounded smooth domain and let $M>0$ be a real number. Let $\mathcal{G}$ be the family of all functions defined in $\Omega$ which are rearrangements of a given function $g_{0}$ with $0 \leq g_{0}(x) \leq M, g_{0}(x) \not \equiv 0, g_{0}(x) \not \equiv M$. For $g \in \mathcal{G}$, let $\lambda_{g}$ be the first eigenvalue of problem (1), and let $\Lambda_{g}$ be the first eigenvalue of problem (2). We investigate the problems

$$
\min _{g \in \mathcal{G}} \lambda_{g}, \quad \text { and } \quad \min _{g \in \mathcal{G}} \Lambda_{g} .
$$

Recalling (3) and (4) we can formulate the previous problems as

$$
\min _{g \in \mathcal{G}} \lambda_{g}=\min \left\{\frac{\int_{\Omega}(\Delta z)^{2} d x}{\int_{\Omega} g z^{2} d x}: g \in \mathcal{G}, z \in H_{0}^{1}(\Omega), \Delta z \in H_{0}^{1}(\Omega)\right\},
$$

and

$$
\min _{g \in \mathcal{G}} \Lambda_{g}=\min \left\{\frac{\int_{\Omega}(\Delta z)^{2} d x}{\int_{\Omega} g z^{2} d x}: g \in \mathcal{G}, z \in H_{0}^{2}(\Omega)\right\} .
$$

Theorem 3.1 Let $0 \leq g_{0}(x) \leq M, g_{0}(x) \not \equiv 0, g_{0}(x) \not \equiv M$, and let $\mathcal{G}$ be the class of all rearrangements of $g_{0}$. Then

a) there exists $\bar{g} \in \mathcal{G}$ such that

$$
\lambda_{\bar{g}}=\min _{g \in \mathcal{G}} \lambda_{g}
$$

b) there exists $\tilde{g} \in \mathcal{G}$ such that

$$
\Lambda_{\tilde{g}}=\min _{g \in \mathcal{G}} \Lambda_{g}
$$

Proof. We prove first part a). Let

$$
I=\inf _{g \in \mathcal{G}} \lambda_{g}=\lim _{i \rightarrow \infty} \lambda_{g_{i}}=\lim _{i \rightarrow \infty} \frac{\int_{\Omega}\left(\Delta u_{i}\right)^{2} d x}{\int_{\Omega} g_{i} u_{i}^{2} d x},
$$

where $u_{i}=u_{g_{i}}$ is the eigenfunction corresponding to $g_{i}$ normalized so that

$$
\int_{\Omega} u_{i}^{2} d x=1
$$

We may assume that the sequence $\left\{\lambda_{g_{i}}\right\}$ is decreasing. By (9) and the latter equation we get

$$
\int_{\Omega}\left(\Delta u_{i}\right)^{2} d x \leq \lambda_{g_{1}} M
$$

On the other side, since $u_{i}$ vanishes on $\partial \Omega$, by Lemma 9.17 of [11] we have

$$
\left\|u_{i}\right\|_{H^{2}(\Omega)} \leq C\left\|\Delta u_{i}\right\|_{L^{2}(\Omega)}
$$

with $C$ independent of $i$. It follows that the norms $\left\|u_{i}\right\|_{H^{2}(\Omega)}$ and $\left\|\Delta u_{i}\right\|_{L^{2}(\Omega)}$ are equivalent. This fact and (10) imply that the sequence $\left\{u_{i}\right\}$ is bounded in the $H^{2}(\Omega)$ norm and some subsequence (still denoted $\left\{u_{i}\right\}$ ) converges weakly in $H^{2}(\Omega)$ to a function $\bar{u}$. We 
can also assume that $\left\{u_{i}\right\}$ converges strongly to $\bar{u}$ in $L^{2+\epsilon}(\Omega)$ for some $\epsilon>0$. Furthermore, since $\left\{g_{i}\right\}$ is bounded in $L^{\infty}(\Omega)$, it must contain a subsequence (still denoted $\left\{g_{i}\right\}$ ) converging weakly to $\eta \in L^{r}(\Omega)$ for any $r>1$. We have

$$
\int_{\Omega} g_{i} u_{i}^{2} d x-\int_{\Omega} \eta \bar{u}^{2} d x=\int_{\Omega}\left(g_{i}-\eta\right) \bar{u}^{2} d x+\int_{\Omega} g_{i}\left(u_{i}^{2}-\bar{u}^{2}\right) d x .
$$

We find

$$
\lim _{i \rightarrow \infty} \int_{\Omega}\left(g_{i}-\eta\right) \bar{u}^{2} d x=0,
$$

because $\bar{u}^{2} \in L^{s}(\Omega)$ for some $s>1$ and $g_{i} \rightarrow \eta$ weakly in $L^{r}(\Omega)$ for $r=s /(s-1)$. Moreover,

$$
\lim _{i \rightarrow \infty} \int_{\Omega} g_{i}\left(u_{i}^{2}-\bar{u}^{2}\right) d x=0 .
$$

The latter result can be proved by using Lebesgue's theorem as follows. Since $u_{i} \rightarrow \bar{u}$ in $L^{2}(\Omega)$, we have (up to a subsequence)

$$
\lim _{i \rightarrow \infty} g_{i}\left(u_{i}^{2}-\bar{u}^{2}\right)=0 \text { a.e. in } \Omega
$$

and

$$
g_{i}\left|u_{i}^{2}-\bar{u}^{2}\right| \leq M\left(\psi^{2}+\bar{u}^{2}\right)
$$

for some integrable function $\psi^{2}$. Indeed, since $u_{i}$ converges in $L^{2}(\Omega)$ one can find $\psi \in L^{2}(\Omega)$ such that $u_{i}(x) \leq \psi(x)$ a.e. for some subsequence of $u_{i}[10]$. Hence,

$$
\lim _{i \rightarrow \infty} \int_{\Omega} g_{i} u_{i}^{2} d x=\int_{\Omega} \eta \bar{u}^{2} d x .
$$

By Lemma 2.3 we can find $\bar{g} \in \mathcal{G}$ such that

$$
\int_{\Omega} \eta \bar{u}^{2} d x \leq \int_{\Omega} \bar{g} \bar{u}^{2} d x .
$$

On the other side, from the inequality

$$
0 \leq \int_{\Omega}\left(\Delta\left(u_{i}-\bar{u}\right)\right)^{2} d x=\int_{\Omega}\left(\Delta u_{i}\right)^{2} d x-2 \int_{\Omega} \Delta u_{i} \Delta \bar{u} d x+\int_{\Omega}(\Delta \bar{u})^{2} d x
$$

and the weak convergence of $\left\{u_{i}\right\}$ to $\bar{u}$ in $H^{2}(\Omega)$ we find

$$
\liminf _{i \rightarrow \infty} \int_{\Omega}\left(\Delta u_{i}\right)^{2} d x \geq \int_{\Omega}(\Delta \bar{u})^{2} d x
$$

By using the latter result together with (11) and (12) we have

$$
I=\lim _{i \rightarrow \infty} \frac{\int_{\Omega}\left(\Delta u_{i}\right)^{2} d x}{\int_{\Omega} g_{i} u_{i}^{2} d x} \geq \frac{\int_{\Omega}(\Delta \bar{u})^{2} d x}{\int_{\Omega} \eta \bar{u}^{2} d x} \geq \frac{\int_{\Omega}(\Delta \bar{u})^{2} d x}{\int_{\Omega} \bar{g} \bar{u}^{2} d x} .
$$


Our minimizing sequence $u_{i}$ satisfies (in a weak sense)

$$
\Delta\left(\Delta u_{i}\right)=\lambda_{g_{i}} g_{i} u_{i}, \quad \Delta u_{i} \in H_{0}^{1}(\Omega)
$$

If we multiply by $-\Delta u_{i}$ and integrate over $\Omega$, after simplification we find

$$
\left\|\nabla\left(\Delta u_{i}\right)\right\|_{L^{2}(\Omega)} \leq \lambda_{g_{i}}\left\|g_{i} u_{i}\right\|_{L^{2}(\Omega)} .
$$

Since $\lambda_{g_{i}}$ is decreasing, $0 \leq g_{i} \leq M$ and $\left\|u_{i}\right\|_{L^{2}(\Omega)}=1$ we find that $\left\|\nabla\left(\Delta u_{i}\right)\right\|_{L^{2}(\Omega)} \leq \lambda_{g_{1}} M$. As a consequence, since $\Delta u_{i} \in H_{0}^{1}(\Omega)$ we also have $\Delta \bar{u} \in H_{0}^{1}(\Omega)$. Therefore, if $\lambda_{\bar{g}}$ is the (first) eigenvalue corresponding to $\bar{g}$ in problem (1), and if $u_{\bar{g}}$ is a corresponding eigenfunction then by (3) we have

$$
\frac{\int_{\Omega}(\Delta \bar{u})^{2} d x}{\int_{\Omega} \bar{g} \bar{u}^{2} d x} \geq \frac{\int_{\Omega}\left(\Delta u_{\bar{g}}\right)^{2} d x}{\int_{\Omega} \bar{g} u_{\bar{g}}^{2} d x}=\lambda_{\bar{g}} \geq I .
$$

By the latter result and (13) we must have $I=\lambda_{\bar{g}}$. Part a) of the theorem is proved.

The proof of part b) is similar. Define

$$
\tilde{I}=\inf _{g \in \mathcal{G}} \Lambda_{g}=\lim _{i \rightarrow \infty} \frac{\int_{\Omega}\left(\Delta v_{i}\right)^{2} d x}{\int_{\Omega} g_{i} v_{i}^{2} d x}
$$

where $v_{i}=v_{g_{i}}$ is the eigenfunction corresponding to $g_{i}$ normalized so that

$$
\int_{\Omega} v_{i}^{2} d x=1
$$

Of course, $\left\{g_{i}\right\}$ is not, in general, the same as for part a). Arguing as in the previous case we find that $v_{i}$ is bounded in the norm of $H^{2}(\Omega)$. Therefore, a subsequence (still denoted $\left\{v_{i}\right\}$ ) converges weakly in $H^{2}(\Omega)$ to a function $\tilde{v} \in H_{0}^{2}(\Omega)$. We can also assume that $\left\{v_{i}\right\}$ converges strongly to $\tilde{v}$ in $L^{2+\epsilon}(\Omega)$ for some $\epsilon>0$. Furthermore, $\left\{g_{i}\right\}$ must contain a subsequence (still denoted $\left\{g_{i}\right\}$ ) converging weakly to some $\zeta \in L^{r}(\Omega)$ for any $r>1$. Hence,

$$
\lim _{i \rightarrow \infty} \int_{\Omega} g_{i} v_{i}^{2} d x=\int_{\Omega} \zeta \tilde{v}^{2} d x
$$

By Lemma 2.3 we can find $\tilde{g} \in \mathcal{G}$ such that

$$
\int_{\Omega} \zeta \tilde{v}^{2} d x \leq \int_{\Omega} \tilde{g} \tilde{v}^{2} d x
$$

Moreover we have

$$
\liminf _{i \rightarrow \infty} \int_{\Omega}\left(\Delta v_{i}\right)^{2} d x \geq \int_{\Omega}(\Delta \tilde{v})^{2} d x
$$

Using the last three results we find

$$
\tilde{I}=\lim _{i \rightarrow \infty} \frac{\int_{\Omega}\left(\Delta v_{i}\right)^{2} d x}{\int_{\Omega} g_{i} v_{i}^{2} d x} \geq \frac{\int_{\Omega}(\Delta \tilde{v})^{2} d x}{\int_{\Omega} \zeta \tilde{v}^{2} d x} \geq \frac{\int_{\Omega}(\Delta \tilde{v})^{2} d x}{\int_{\Omega} \tilde{g} \tilde{v}^{2} d x} .
$$


Recall that $\tilde{v} \in H_{0}^{2}(\Omega)$ and $\tilde{g} \in \mathcal{G}$. If $\Lambda_{\tilde{g}}$ is the (first) eigenvalue corresponding to $\tilde{g}$ in problem (2), and if $v_{\tilde{g}}$ is a corresponding eigenfunction then, using (4) we have

$$
\frac{\int_{\Omega}(\Delta \tilde{v})^{2} d x}{\int_{\Omega} \tilde{g} \tilde{v}^{2} d x} \geq \frac{\int_{\Omega}\left(\Delta v_{\tilde{g}}\right)^{2} d x}{\int_{\Omega} \tilde{g} v_{\tilde{g}}^{2} d x}=\Lambda_{\tilde{g}} \geq \tilde{I} .
$$

By (14) and (15) we must have $\tilde{I}=\Lambda_{\tilde{g}}$. The theorem is proved.

We prove the so called Euler-Lagrange equation for solutions of our minimization problems. Actually, there is a difference between the two cases. Concerning problem (1), we know that the first eigenfunction does not change sign, and we can assume that it is positive in $\Omega$. Concerning problem (2), there are domains $\Omega$ such that the corresponding first eigenfunction is sign changing, and there are domains such that the corresponding first eigenfunction is positive: see [12] and references therein.

In what follows we write $\{g(x)>0\}$ instead of $\{x \in \Omega: g(x)>0\}$.

Theorem 3.2 a) Suppose $\bar{g}$ is a solution to problem (7). There exists an increasing function $\phi$ such that

$$
\bar{g}=\phi\left(u_{\bar{g}}\right) .
$$

b) Suppose $\bar{g}$ is a solution to problem (8) and that $\Omega$ is such that the corresponding first eigenfunction of problem (2) is positive. There exists an increasing function $\varphi$ such that

$$
\bar{g}=\varphi\left(u_{\bar{g}}\right)
$$

Proof. If $u_{\bar{g}}$ is the positive normalized eigenfunction corresponding to the minimizer $\bar{g}$ of problem (7), for any $g \in \mathcal{G}$ we have

$$
\frac{\int_{\Omega}\left(\Delta u_{\bar{g}}\right)^{2} d x}{\int_{\Omega} \bar{g} u_{\bar{g}}^{2} d x} \leq \frac{\int_{\Omega}\left(\Delta u_{\bar{g}}\right)^{2} d x}{\int_{\Omega} g u_{\bar{g}}^{2} d x} .
$$

Hence,

$$
\int_{\Omega} g u \frac{2}{g} d x \leq \int_{\Omega} \bar{g} u \frac{2}{g} d x
$$

for all $g \in \mathcal{G}$.

On the other side, we know that the function $u_{\bar{g}}$ satisfies the eigenvalue equation

$$
\Delta^{2} u_{\bar{g}}=\lambda \bar{g} u_{\bar{g}}
$$

If $-\Delta u_{\bar{g}}=v$, by the above equation we have $-\Delta v \geq 0$ in $\Omega$ and $v=0$ on $\partial \Omega$. It follows that $v(x)>0$ in $\Omega$. Since $-\Delta u_{\bar{g}}>0$, the function $u_{\bar{g}}$ cannot have level sets of positive measure. Hence, by Lemma 2.1, inequality (16) and Lemma 2.2 we infer the existence of an increasing function $\phi_{1}$ such that $\bar{g}=\phi_{1}\left(u_{\bar{g}}^{2}\right)$. Thus, part a) of the theorem follows with $\phi(t)=\phi_{1}\left(t^{2}\right)$.

If $u_{\bar{g}}$ is the positive normalized eigenfunction corresponding to the minimizer $\bar{g}$ of problem (8), inequality (16) holds for all $g \in \mathcal{G}$. Moreover, $\Delta^{2} u_{\bar{g}}=\Lambda \bar{g} u_{\bar{g}}$. By this equation, the function $u_{\bar{g}}$ cannot have level sets of positive measure on $\{\bar{g}(x)>0\}$. If the 
set $\{\bar{g}(x)=0\}$ has zero measure, by Lemma 2.1, inequality (16) and Lemma 2.2 we infer the existence of an increasing function $\varphi_{1}$ such that $\bar{g}=\varphi_{1}\left(u_{\bar{g}}^{2}\right)$. Thus, in this case part b) of the theorem follows with $\varphi(t)=\varphi_{1}\left(t^{2}\right)$. Otherwise, setting $E=\{\bar{g}(x)=0\}$, we define:

$$
S=\sup _{x \in E}\left(u_{\bar{g}}(x)\right)^{2} .
$$

By using (16) one proves that $\left(u_{\bar{g}}(x)\right)^{2} \geq S$ on $\{\bar{g}(x)>0\}$ a.e. For the proof of this result we refer to [8], Theorem 3.2. Since $u \bar{g}$ cannot have level sets of positive measure on $\Omega \backslash E$, by Lemma 2.1 we infer the existence of an increasing function $\varphi_{1}:(S, \infty) \rightarrow[0, M]$ such that $\varphi_{1}\left(u_{\bar{g}}^{2}\right)$ is a rearrangement of $\bar{g}$ on $\Omega \backslash E$. Now we define an increasing function $\varphi_{2}$ as

$$
\varphi_{2}(t)= \begin{cases}0 & t \leq S \\ \varphi_{1}(t) & t>S\end{cases}
$$

Since $\varphi_{2}\left(u_{\bar{g}}^{2}\right)$ is a rearrangement of $\bar{g}$ on $\Omega$, by inequality (16) and Lemma 2.2 we infer that $\bar{g}=\phi_{2}\left(u_{\bar{g}}^{2}\right)$. Part b) of the theorem follows taking $\varphi(t)=\varphi_{2}\left(t^{2}\right)$. The theorem is proved.

Remarks. Theorem 3.2 gives some information on the location of the materials in order to minimize the first eigenvalue of problem (7). Indeed, since the associate eigenfunction $u_{\bar{g}}$ vanishes on the boundary $\partial \Omega$, and $\bar{g}=\phi\left(u_{\bar{g}}\right)$ with $\phi$ increasing, the material with higher density must be located where $u_{\bar{g}}$ is large, that is, far from $\partial \Omega$. The same remark holds for problem (8) in appropriate domains.

Theorem 3.3 Let $B$ be a ball in $\mathbb{R}^{N}$, and let $g$ be a minimizer of either problem (7) or problem (8) with $\Omega=B$. Then $g=g^{\sharp}$.

Proof. If $g$ is a minimizer of problem (7) and if $u=u_{g}$ is a corresponding positive eigenfunction we have

$$
\lambda_{g}=\frac{\int_{B}(\Delta u)^{2} d x}{\int_{B} g u^{2} d x} .
$$

Put

$$
-\Delta u=z
$$

Then

$$
-\Delta z=\lambda_{g} g u
$$

Since $u>0$ in $B$ and $z=0$ on $\partial B$ we have $z>0$ in $B$. If $z^{\sharp}$ is the Schwarz decreasing rearrangement of $z$ then $z^{\sharp} \in H_{0}^{1}(B)$ and

$$
\int_{B}(\Delta u)^{2} d x=\int_{B}\left(z^{\sharp}\right)^{2} d x .
$$

Furthermore, if $\bar{u}$ is the solution to the problem

$$
-\Delta \bar{u}=z^{\sharp} \text { in } B, \quad \bar{u}=0 \text { on } \partial B
$$


then, by a result of G. Talenti ([15], Theorem 1, (iv)) we have

$$
u^{\sharp} \leq \bar{u} \text { in } B \text {. }
$$

By a well known inequality on rearrangements and (21) we find

$$
\int_{B} g u^{2} d x \leq \int_{B} g^{\sharp}\left(u^{\sharp}\right)^{2} d x \leq \int_{B} g^{\sharp}(\bar{u})^{2} d x .
$$

Since $\left(z^{\sharp}\right)^{2}=(\Delta \bar{u})^{2}$, by (19), (17) and (22) we find

$$
\lambda_{g} \geq \frac{\int_{B}(\Delta \bar{u})^{2} d x}{\int_{B} g^{\sharp}(\bar{u})^{2} d x} \geq \frac{\int_{B}\left(\Delta u_{g^{\sharp}}\right)^{2} d x}{\int_{B} g^{\sharp}\left(u_{g^{\sharp}}\right)^{2} d x}=\lambda_{g^{\sharp}} .
$$

In the last step we have used the fact that $\bar{u}$ is admissible (because $\bar{u}=\Delta \bar{u}=0$ on $\partial B$ ) and the variational characterization of $\lambda_{g^{\sharp}}$. Since $\lambda_{g}$ is a minimizer, we must have $\lambda_{g^{\sharp}}=\lambda_{g}$ and equality must hold in (22). In particular,

$$
\int_{B} g^{\sharp}\left(u^{\sharp}\right)^{2} d x=\int_{B} g^{\sharp}(\bar{u})^{2} d x .
$$

Recalling that $g(x)$ is positive in a set of positive measure we have $g^{\sharp}(x)>0$ in a ball $B\left(r_{0}\right)$ of radius $r_{0}$ for some $r_{0}>0$. Therefore the previous equation and (21) imply that $u^{\sharp}(0)=\bar{u}(0)$. An inspection of the proof of Talenti's result [15] (see also [2]) yields $u^{\sharp}(x)=\bar{u}(x)$ in all of $B$. Moreover by (18), (20) with $\bar{u}=u^{\sharp}$, and (6) we find

$$
\int_{B}|\nabla u|^{2} d x=\int_{B} u z d x \leq \int_{B} u^{\sharp} z^{\sharp} d x=\int_{B}\left|\nabla u^{\sharp}\right|^{2} d x \leq \int_{B}|\nabla u|^{2} d x .
$$

It follows that

$$
\int_{B}|\nabla u|^{2} d x=\int_{B}\left|\nabla u^{\sharp}\right|^{2} d x .
$$

By Lemma 2.4 we get $u(x)=u^{\sharp}(x)$ in $B$. Furthermore, by Theorem 3.2 a) we have $g=\phi(u)$ for some increasing function $\phi$. This implies that $g$ is radially symmetric and decreasing, hence $g=g^{\sharp}$. The theorem is proved in this case.

Let us come to problem (8). Putting $-\Delta v=w$ and recalling that $\frac{\partial v}{\partial \nu}=0$ on $\partial B$ we find

$$
\int_{B} w d x=-\int_{B} \Delta v d x=\int_{\partial B} \frac{\partial v}{\partial \nu} d \sigma=0
$$

This means that $w(x)$ is sign changing in $B$. Let $w^{\sharp}(x)$ be the signed Schwarz decreasing rearrangement of $w(x)$ and let

$$
-\Delta \bar{v}=w^{\sharp} \text { in } B, \quad \bar{v}=0 \text { on } \partial B .
$$

Since $\int_{B} w^{\sharp} d x=0$ the result of Talenti [15] continues to hold as observed also in [2]. Hence,

$$
v^{\sharp} \leq \bar{v} \text { in } B .
$$

Moreover, since

$$
0=-\int_{B} w d x=-\int_{B} w^{\sharp} d x=\int_{B} \Delta \bar{v} d x=\int_{\partial B} \frac{\partial \bar{v}}{\partial \nu} d \sigma=\frac{\partial \bar{v}}{\partial \nu}|\partial B|,
$$

we have $\bar{v} \in H_{0}^{2}(B)$. The proof continues as in the previous case. 


\section{References}

[1] Agmon, S.; Douglis, A.; Nirenberg, L. (1959) "Estimates near the boundary for solutions of elliptic partial differential equations satisfying general boundary conditions. I," Commun. Pure Appl. Math., 12: 623-727.

[2] Anedda, C. (2008) "Maximization and minimization in problems involving the biLaplacian", Preprint N. 1, Maths Department, Univ. Cagliari, Italy: 1-12.

[3] Brothers, J.E.; Ziemer, W.P. (1988) "Minimal rearrangements of Sobolev functions", J. Reine Angew. Math., 384: 153-179.

[4] Burton, G.R. (1989) "Variational problems on classes of rearrangements and multiple configurations for steady vortices", Ann. Inst. Henri Poincaré, 6(4): 295-319.

[5] Burton, G.R.; McLeod, J.B. (1991) "Maximisation and minimisation on classes of rearrangements", Proc. Roy. Soc. Edinburgh Sect. A, 119(3-4): 287-300.

[6] Chanillo, S.; Grieser, D.; Imai, M.; Kurata, K.; Ohnishi, I. (2000) "Symmetry breaking and other phenomena in the optimization of eigenvalues for composite membranes", Commun. Math. Phys., 214: 315-337.

[7] Cox, S.J.; McLaughlin, J.R. (1990) "Extremal eigenvalue problems for composite membranes, I, II", Appl. Math. Optim., 22: 153-167; 169-187.

[8] Cuccu, F.; Emamizadeh, B.; Porru, G. (s.f.) "Optimization of the first eigenvalue in problems involving the p-Laplacian", Proc. Amer. Math. Soc. To appear.

[9] Cuccu, F.; Porru, G. (2003) "Optimization in eigenvalue problems", Dyn. Contin. Discrete Impuls. Syst. Ser. A Math. Anal., 10: 51-58.

[10] Evans, L.; Gariepy, R.F. (1992) Measure Theory and Fine Properties of Functions. Studies in Advanced Mathematics. CRC Press, Boca Raton.

[11] Gilbarg, D.; Trudinger, N.S. (1977) Elliptic Partial Differential Equations of Second Order. Springer Verlag, Berlin.

[12] Grunau, H.C.; Sweers, G. (1999) "Sign change for the Green function and for the first eigenfunction of equations of clamped-plate type", Arch. Ration. Mech. Anal. 150: $179-190$.

[13] Kawohl, B. (1985) Rearrangements and Convexity of Level Sets in PDE. Lectures Notes in Mathematics, 1150, Berlin.

[14] Struwe, M. (1990) Variational Methods. Springer-Verlag, Berlin, New York.

[15] Talenti, G. (1976) "Elliptic equations and rearrangements", Ann. Sc. Norm. Sup. Pisa, Ser. IV, 3: 697-718. 